\title{
Síntesis de nuevos pigmentos cerámicos de color rojo mediante el método de Pechini
}

\author{
R.PAVLOV, V. BLASCO, E. CORDONCILLO, P. ESCRIBANO Y J. B. CARDA \\ Departamento de Química Inorgánica y Orgánica. E.S.T.C.E. \\ Universitat Jaume I, 12080 Castellón (España).
}

\begin{abstract}
A través del presente trabajo, se han estudiado la obtención de diferentes disoluciones sólidas en la estructura granate de itrio y aluminio, $\mathrm{Y}_{3} \mathrm{Al}_{5} \mathrm{O}_{12}(\mathrm{YAG})$ sintetizadas vía Pechini y la posibilidad de modificar la intensidad del campo cristalino, $\mathrm{D}_{\mathrm{o}^{\prime}}$ que actúa sobre el ion $\mathrm{Cr}(\mathrm{III})$, como agente cromóforo, mediante la introducción de cationes divalentes (Mn(II) y $\mathrm{Mg}(\mathrm{II})$ ) y tetravalentes (Ce(IV), $\mathrm{Zr}(\mathrm{IV})$ y $\mathrm{Sn}(\mathrm{IV})$ ). Los resultados alcanzados han posibilitado comprender que con la introducción de los iones divalentes, éstos no han sido suficientes para intensificar dicho campo del Cr(III), ya que estabilizan al ion $\mathrm{Cr}$ (IV) y esto provoca distorsiones estructurales. Con la introducción de los iones tetravalentes, sí que se consigue intensificar el campo que actúa sobre el ion cromóforo, observándose la transición de la tonalidad verde, típica del Cr(III) en un campo débil, a una tonalidad roja, típica para el mismo ion en un campo fuerte. Sin embargo y a través de los estudios estructurales mediante difracción de rayos $X$, se ha observado que junto con el desarrollo de la estructura granate, disolución sólida, aparecen fases laterales de $\mathrm{Y}_{0,15} \mathrm{Zr}_{0,85} \mathrm{O}_{1,93}$ y $\mathrm{Y}_{2} \mathrm{Sn}_{2} \mathrm{O}_{7}$, esta última como fase pirocloro. Se ha comprobado la obtención de una tonalidad roja-violácea con la introducción de $\mathrm{Cr}(\mathrm{III})$ en dicha estructura pirocloro. Por último, se han probado las fases sintetizadas como pigmentos mediante la utilización de un vidriado (frita) transparente de naturaleza borácica, industrial. Los materiales que contienen estaño en su estructura han mostrado un buen poder pigmentante y estabilidad a $1080^{\circ} \mathrm{C}$.
\end{abstract}

Palabras clave: granates, pigmentos cerámicos, Pechini, síntesis, cristaloquímica.

Synthesis of new red coloured ceramic pigments by Pechini method

Various garnet solid solutions within the structure of the yttrium aluminium garnet, $\mathrm{Y}_{3} \mathrm{Al}_{5} \mathrm{O}_{12}(\mathrm{YAG})$ were sinthesized via Pechini and the possibility for the modification of the crystal field intensity acting on the chromophore $\mathrm{Cr}$ (III) ion by means of substitution with divalent (Mn(II) and $\mathrm{Mg}(\mathrm{II})$ ) and tetravalent (Ce(IV), $\mathrm{Zr}(\mathrm{IV})$ and $\mathrm{Sn}(\mathrm{IV})$ ) cations was studied. The results obtained indicate that the crystal field can not be intensifyed through the introduction of divalent ions since they stabilize the $\mathrm{Cr}(\mathrm{IV})$ ion which probably expands the structure by inducing distortions in it. The introduction of tetravalent ions makes possible the intensification of the crystal field acting on the chromium and the change of the typical green colour of $\mathrm{Cr}(\mathrm{III})$ in a week field to red which is typical for the same in strong field. However, the structural studies carried out by $\mathrm{X}$-ray diffraction show that together with the garnet fase $\mathrm{Y}_{015} \mathrm{Zr}_{0.85} \mathrm{O}_{1,93}$ and $\mathrm{Y}_{2} \mathrm{Sn}_{2} \mathrm{O}_{7}$ are formed, the latter having pyrochlore structure. It was demonstarted that the introduction of $\mathrm{Cr}$ (III) in the mantioned pyrochlore structure results in the formation of a redviolet pigment. Finally, the sinthesized fases were checked as pigments using an industrial transparent boron containing frit. The tin containing pigments demonstrated good pigmenting ability and stability at $1080^{\circ} \mathrm{C}$.

Keywords: garnets, ceramic pigments, Pechini, synthesis, crystalchemistry

\section{INTRODUCCIÓN}

La industria española de pavimentos y revestimientos cerámicos ha manifestado un gran auge en los últimos años, posibilitado por el volumen de su producción, gracias al alto grado de automatización de los procesos y por el desarrollo de productos más tecnológicos. En este sentido, el gres porcelánico es un buen ejemplo de ello, lo que implica unos ciclos de producción más extremos (1).

Paralelamente, se están implantando también nuevas tecnologías en el campo de la decoración que exigen nuevos productos más acordes para su aplicación. En esta línea, es de gran actualidad la búsqueda de nuevos pigmentos cerámicos que sean estables a los ciclos productivos que se llevan a cabo en el sector industrial de pavimentos y revestimientos cerámicos y que sean compatibles con los vidriados en los que se ven inmersos es de gran actualidad. Por otra parte, con el desarrollo de nuevas pastas cerámicas, que dan origen a productos más tecnológicos, como es el caso del desarrollo de pastas de gres porcelánico, se exige disponer de nuevos pigmentos que den origen a tonalidades más intensas y acordes a estos nuevos ciclos productivos (2). Una de las tonalidades de mayor exigencia y de gran interés en el campo de la investigación es la obtención de colores rojos estables a altas temperaturas y compatibles con las exigencias medioambientales (3) (4). En este sentido, existe en el sector productivo un pigmento rojo de sulfoseleniuro de cadmio, $\mathrm{CdSe}_{\mathrm{x}} \mathrm{S}_{1-\mathrm{x}^{\prime}}$ estabilizado con la estructura de circón, (5), pero con la problemática en su proceso de fabricación por la manipulación de estos materiales tan tóxicos. 
Una de las estructuras candidatas para ser moduladas en su composición por su amplia variedad cristaloquímica y su estabilidad térmica, es la estructura del granate (6). Los granates se describen estructuralmente bajo la fórmula $\mathrm{A}_{3} \mathrm{~B}_{2} \mathrm{C}_{3} \mathrm{O}_{12}$ donde $\mathrm{A}$ suele representar a un catión divalente en coordinación octaédrica, B representa a un catión trivalente en coordinación octaédrica y $\mathrm{C}$ a un catión tetravalente en coordinación tetraédrica. La estructura presenta una simetría cúbica, con el grupo espacial Ia3d.

Existen descritos pigmentos cerámicos con esta estructura. Uno de ellos, comercializado industrialmente, es el pigmento "Verde Victoria" con la estructura del granate uvarovita, $\mathrm{Ca}_{3} \mathrm{Cr}_{2} \mathrm{Si}_{3} \mathrm{O}_{12}(7,8,9)$. Sin embargo, se ha observado que la fuerte rigidez que presenta el enlace $\mathrm{Si}-\mathrm{O}$, por su marcado carácter covalente, no permite distorsionar suficientemente la estructura e imposibilita obtener fácilmente disoluciones sólidas con dicha estructura (10). De hecho, el granate de silicato de calcio y cromo es de los pocos granates sintetizados a presión atmosférica. Una posibilidad de flexibilizar la rigidez estructural que presentan los granates silicatos es mediante la introducción de cationes de mayor tamaño en las posiciones tetraédricas que viene ocupando el catión Si (IV).

En este sentido y a través del presente trabajo, se ha elegido la estructura del granate de itrio y aluminio, $\mathrm{Y}_{3} \mathrm{Al}_{5} \mathrm{O}_{12}$, conocido como YAG, por presentar unas propiedades de alta resistencia mecánica, térmica y química y la flexibilidad de su estructura, lo que permite modificar sus propiedades en un amplio rango. Un ejemplo de ello es su aplicación en el campo de la óptica y el tradicional desarrollo de láseres y fósforos en esta estructura $(11,12,13,14)$.

La particularidad que presenta dicha estructura, es la de la ocupación simultánea de las posiciones tetraédricas y octaédricas por el ion $\mathrm{Al}(\mathrm{III})$. El itrio, de mayor tamaño, se encuentra ocupando las posiciones dodecaédricas.

Por otra parte hay que indicar la importancia que tiene el Cr(III) para su utilización como elemento cromóforo ya que presenta unas interesantes propiedades ópticas derivadas de sus espectros electrónicos (15)

En la estructura del granate YAG el cromo(III), así como también el cromo(IV) se han estudiado siempre bajo el punto de vista del desarrollo y mejora de los medios activos de láseres y poca atención se ha prestado a sus propiedades cromáticas. El cromo(III) por su tamaño puede sustituir únicamente al aluminio en las posiciones octaédricas. Existe también la posibilidad de la realización de sustitución isomórfica de aluminio tetraédrico por cromo. Esto puede ocurrir si el cromo(III) se oxida a cromo(IV), cuyo radio iónico es muy parecido al del aluminio tetraédrico. En general, para estabilizar el cromo(IV) se introducen compensadores de carga, tales como $\mathrm{Ca}$ (II) o $\mathrm{Mg}$ (II) pero aún en el mejor caso sólo un $2 \%$ de todo el cromo introducido en la estructura está en estado de oxidación cuatro (16).

El estado fundamental de un ion $\mathrm{d}^{3}$ como es el $\mathrm{Cr}^{3+}$, es ${ }^{4} \mathrm{~A}_{2 \mathrm{~g}}$ (17) Las transiciones de este estado a los estados ${ }^{2} \mathrm{E}_{\mathrm{g}^{\prime}}{ }^{2} \mathrm{~T}_{1 \mathrm{~g}} \mathrm{y}$ ${ }^{2} \mathrm{~T}_{2 \mathrm{~g}}$ están prohibidas por spin. La primera transición permitida es del estado ${ }^{4} \mathrm{~A}_{2 \mathrm{~g}}$ al estado ${ }^{4} \mathrm{~T}_{2 \mathrm{~g}} \mathrm{y}$, la diferencia entre las correspondientes energías, determina el valor del parámetro del campo cristalino. Esta transición y la siguiente permitida, de ${ }^{4} \mathrm{~A}_{2 \mathrm{~g}}$ a ${ }^{4} \mathrm{~T}_{1 \mathrm{~g}}$, dan lugar a dos amplias bandas en los espectros de absorción en el visible, cuyas posiciones y forma determinan el color del complejo. El mínimo entre las dos bandas corresponde a la reflexión en azul, verde o amarillo, según la fuerza del campo. El umbral de baja energía de la banda debida a la transición ${ }^{4} \mathrm{~A}_{2 \mathrm{~g}} \rightarrow{ }^{4} \mathrm{~T}_{2 \mathrm{~g}^{\prime}}$ puede caer fuera del espectro visible. Esto ocurre en el caso de un campo débil y entonces la tonalidad desarrollada es verde, siendo determinada sólo por la región comprendida entre los máximos de las dos bandas. Si dicho umbral está desplazado hacia longitudes de onda más cortas y, el mínimo entre las dos bandas está poco profundo y en el azul, la tonalidad correspondiente es roja o rojaviolácea. Esto ocurre cuando el cromo(III) se encuentra en un campo fuerte.

La magnitud del campo cristalino está relacionada directamente con la distancia metal-oxígeno en el complejo octaédrico, siendo valida la siguiente relación $(18,19)$ :

$$
\Delta_{0}=\text { constante } / \mathrm{d}(\mathrm{Me}-\mathrm{O})^{5},
$$

En la que $\Delta_{0}$ es el parámetro del campo cristalino, el cual en el caso de iones $\mathrm{d}^{3}$ equivale a la energía de la transición ${ }^{4} \mathrm{~A}_{2 \mathrm{~g}} \rightarrow{ }^{4} \mathrm{~T}_{2 \mathrm{~g}}$. $\mathrm{d}(\mathrm{Me}-\mathrm{O})$ es el valor de la distancia metal-oxígeno en coordinación octaédrica. La disminución de esta distancia proporcionará un aumento de la energía del campo cristalino que actúa sobre el cromo y, por consiguiente, una transición de verde a rojo.

Por otra parte la presencia de cromo(IV) aún en las bajas concentraciones en que ocurre en los sistemas óxidos puede afectar mucho a las propiedades cromáticas, ya que tiene una absorción especifica muy elevada. Como un ion $\mathrm{d}^{2}$ en campo tetraédrico ( ) tiene un estado fundamental ${ }^{3} \mathrm{~A}_{2}$. Las transiciones de este estado a los estados excitados ${ }^{3} \mathrm{~T}_{1} \mathrm{y}^{3} \mathrm{~T}_{2}$ son permitidas por spin y dan origen a dos intensas bandas de absorción. La banda que corresponde a la transición ${ }^{3} \mathrm{~A}_{2} Æ^{3} \mathrm{~T}_{2}$ en el granate YAG normalmente se sitúa en el infrarrojo cercano y tiene el máximo aproximadamente a $1000 \mathrm{~nm}$. La transición ${ }^{3} \mathrm{~A}_{2} \rightarrow{ }^{3} \mathrm{~T}_{2}$ aparece como una banda en el visible entre 600 y 700 $\mathrm{nm}$. A esta última se debe la tonalidad marrón de sistemas con altas concentraciones de $\mathrm{Cr}(\mathrm{IV})$ (20)

Teóricamente, el simple hecho de disminuir la concentración del cromo debería producir un aumento en el campo cristalino dado el radio iónico del cromo(III) que es menor que el del aluminio(III) en coordinación octaédrica. Esto se ha observado por ejemplo en el rubí (21). Asimismo la sustitución de cualquier ion en cualquiera de las tres posiciones $\mathrm{A}, \mathrm{B}$ o $C$ por otro ion más pequeño, debería proporcionar la contracción de toda la red cristalina, aumentando así el campo electrostático que actúa sobre el cromo hexacoordinado y, por tanto, la energía de sus transiciones electrónicas.

Así, el objetivo planteado en el presente estudio es el desarrollo de pigmentos cerámicos de tonalidad roja, estables, a partir de la modulación de la estructura del granate YAG, mediante la introducción de cationes divalentes [Mn(II) y $\mathrm{Mg}(\mathrm{II})]$ y tetravalentes [Ce(IV), $\mathrm{Zr}(\mathrm{IV})$ y $\mathrm{Sn}(\mathrm{IV})]$, introduciendo también en la misma el catión Cr(III), como elemento cromóforo, estudiando así la modificación del campo cristalino de dicho cromóforo en las disoluciones sólidas, sintetizadas por vía Pechini $(22,23)$.

\section{PARTE EXPERIMENTAL}

\subsection{Materiales y métodos}

En la tabla I se indican las composiciones nominales preparadas, cuyos precursores se indican en la tabla II.

Para la síntesis de dichos materiales se ha utilizado el método de Pechini, tal y como se ilustra en la figura 1. Así, los correspondientes precursores han sido pesados todos ellos en 
una balanza analítica y han sido todos ellos disueltos previamente en agua, en la proporción de $50 \mathrm{ml}$ por cada gramo de la composición nominal del granate. Seguidamente se han añadido las correspondientes cantidades de ácido cítrico (AC) y etilenglicol (EG), siendo la proporción molar elegida por cada mol de ion metálico de: $\left[\mathrm{Me}^{+}\right]:[\mathrm{AC}]:[\mathrm{EG}]=1: 1: 2$, respectivamente.

Dado que se trabaja con sales precursoras que son muy higroscópicas, se han realizado los correspondientes análisis termogravimétricos de dichas sales, con el fin de garantizar la estequiometría prefijada.

Las disoluciones se han mantenido en continua agitación durante 3 horas a $70^{\circ} \mathrm{C}$ a través de un baño termoestatado de aceite, con el fin de acelerar la reacción y dar lugar a la formación del correspondiente compuesto quelato $(24,25)$. A continuación, las muestras se han dejado secar bajo lámpara de infrarrojos, manteniendo la agitación el tiempo necesario en que la viscosidad del medio lo ha permitido, durante el proceso de densificación. En este sentido, se ha procurado disponer una distancia tal entre la lámpara de infrarrojos y la muestra, para que mantuviera la temperatura constante y próxima a $70^{\circ} \mathrm{C}$. De esta forma se ha llevado a cabo un calentamiento uniforme, acompañado de la evaporación de agua y provocando así la polimerización del sistema. Este proceso ha sido acompañado por la emisión de gases nitrosos $\left(\mathrm{N}_{\mathrm{n}} \mathrm{O}_{\mathrm{m}}\right)$, procedentes de la descomposición de los nitratos de las sales precursores.

Para la síntesis de las disoluciones sólidas $\mathrm{Y}_{2} \mathrm{Sn}_{2-\mathrm{y}} \mathrm{Cr}_{\mathrm{y}} \mathrm{O}_{7} \mathrm{y}$ $\mathrm{Y}_{0.15} \mathrm{Zr}_{0.85-\mathrm{y}} \mathrm{Cr}_{\mathrm{y}} \mathrm{O}_{1.93}$ se ha empleado también el método cerámico. En este caso, como precursores se han usado $\mathrm{Y}_{2} \mathrm{O}_{3}, \mathrm{SnO}_{2}$ y $\mathrm{ZrO}_{2}$ (todos ellos de calidad industrial). Los óxidos tomados en las correspondientes proporciones se han homogeneizado en un molino planetario de bolas en jaras de ágata utilizando acetona como medio de dispersión.

\subsection{Tratamientos térmicos}

Todos los geles crudos obtenidos, una vez secos, han sido acondicionados en crisoles de mullita y han sido sometidos a ciclos de calcinación al aire mediante un horno eléctrico Carbolite, con temperatura máxima de trabajo de $1500^{\circ} \mathrm{C}$. La velocidad de calentamiento ha sido de $4^{\circ} \mathrm{C} / \mathrm{min}$., en el intervalo de temperaturas entre 600 y $1400^{\circ} \mathrm{C}$, con una retención de 4 horas a la tempera máxima y enfriamiento posterior libre.

\subsection{Técnicas de caracterización}

Las principales técnicas de caracterización empleadas en este estudio han sido:

-difracción de rayos $\mathrm{X}$ (DRX), a través de muestras en polvo distribuido al azar, mediante un equipo SIEMENS, modelo D5000, con una geometría de Bragg-Bretano, provisto de cátodo de cobre y filtro de níquel.

-espectrofotometría ultravioleta y visible ( NUV / VIS / NIR), trabajando en modo de reflectancia difusa, a través de un equipo PERKIN ELMER, modelo Lambda 19, midiendo en el intérvalo de 190 a $1200 \mathrm{~nm}$, de longitudes de onda.

-espectrofotometría infrarroja, mediante un equipo PERKIN ELMER, modelo 2000 FT-IR trabajando con muestras prensadas en pastilla de $\mathrm{KBr}$, en el rango de frecuencias de 270 a 4000 $\mathrm{cm}^{-1}$.
TABLA I SERIES DE LAS MUESTRAS PREPARADAS Y SUS COMPOSICIONES NOMINALES

\begin{tabular}{|c|c|}
\hline Fórmula general & $\begin{array}{l}\text { Composiciones nominales de las } \\
\text { muestras preparadas }\end{array}$ \\
\hline $\mathrm{Y}_{3} \mathrm{Al}_{5-\mathrm{y}} \mathrm{Cr}_{\mathrm{y}} \mathrm{O}_{12}$ & $\begin{array}{l}\mathrm{y}=0.05 \\
\mathrm{y}=0.10 \\
\mathrm{y}=0.15 \\
\mathrm{y}=0.20\end{array}$ \\
\hline $\mathrm{Y}_{3-\mathrm{x}}(\mathrm{M}, \mathrm{N})_{\mathrm{x}} \mathrm{Al}_{5-\mathrm{y}} \mathrm{Cr}_{\mathrm{y}} \mathrm{O}_{12}$ & $\begin{array}{c}\mathrm{Y}_{2.95} \mathrm{Mn}_{0.05} \mathrm{Al}_{4.9} \mathrm{Cr}_{0.1} \mathrm{O}_{12} \\
\mathrm{Y}_{2.9} \mathrm{Mg}_{0.1} \mathrm{Al}_{4.9} \mathrm{Cr}_{0.1} \mathrm{O}_{12} \\
\mathrm{Y}_{2.93} \mathrm{Mg}_{0.04} \mathrm{Ca}_{0.03} \mathrm{Al}_{4.98} \mathrm{Cr}_{0.02} \mathrm{O}_{12} \\
\mathrm{Y}_{2.8} \mathrm{Mg}_{0.1} \mathrm{Ce}_{0.1} \mathrm{Al}_{4.8} \mathrm{Cr}_{0.2} \mathrm{O}_{12} \\
\end{array}$ \\
\hline $\mathrm{Y}_{3-\mathrm{x}} \mathrm{Zr}_{\mathrm{x}} \mathrm{Al}_{5-\mathrm{y}} \mathrm{Cr}_{\mathrm{y}} \mathrm{O}_{12+\delta}$ & $\begin{array}{ll}x=0.2 & y=0.1 \\
x=0.4 & y=0.2 \\
x=0.8 & y=0.4 \\
x=0.8 & y=0.1 \\
x=1 & y=0.2\end{array}$ \\
\hline $\mathrm{Y}_{3-\mathrm{x}} \mathrm{Sn}_{\mathrm{x}} \mathrm{Al}_{5-\mathrm{y}} \mathrm{Cr}_{\mathrm{y}} \mathrm{O}_{12+\delta}$ & $x=1 \quad y=0.2$ \\
\hline $\mathrm{Y}_{2} \mathrm{Sn}_{2-\mathrm{y}} \mathrm{Cr}_{\mathrm{y}} \mathrm{O}_{7}$ & $\begin{array}{l}y=0.02 \\
y=0.05 \\
y=0.10 \\
y=0.20\end{array}$ \\
\hline
\end{tabular}

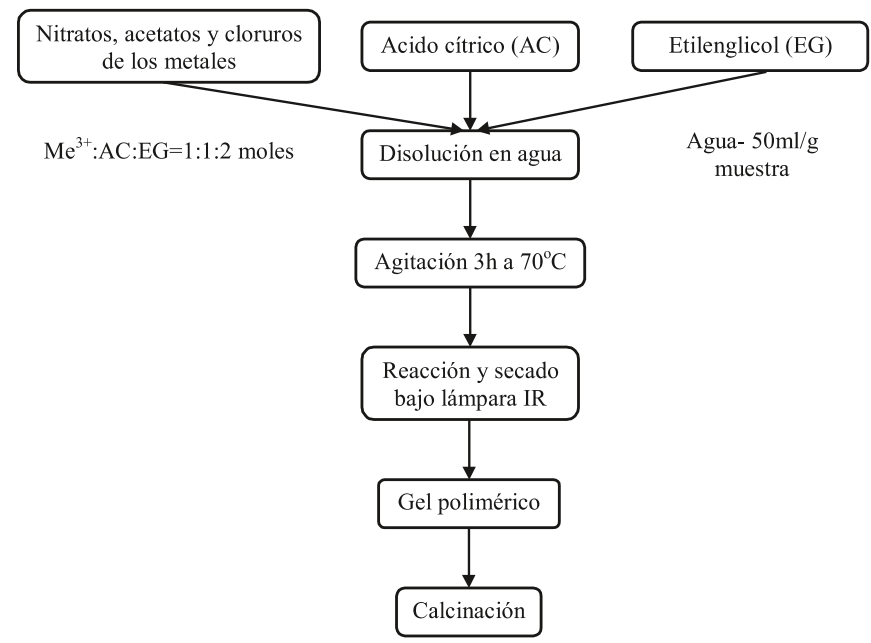

Fig. 1. Procedimiento experimental basado en el método de Pechini.

Tabla II Precursores utilizados en la síntesis vía PechinI.

\begin{tabular}{|c|c|c|}
\hline Óxido & Precursor & Características \\
\hline $\mathrm{Y}_{2} \mathrm{O}_{3}$ & $\mathrm{Y}\left(\mathrm{CH}_{3} \mathrm{COO}\right)_{3} \cdot \mathrm{H}_{2} \mathrm{O}$ & $\begin{array}{l}\text { Sintetizado en nuestro laboratorio a partir } \\
\text { de } \mathrm{Y}_{2} \mathrm{O}_{3} \text {, calidad industrial }\end{array}$ \\
\hline $\mathrm{Al}_{2} \mathrm{O}_{3}$ & $\begin{array}{c}\mathrm{Al}\left(\mathrm{NO}_{3}\right)_{3} \cdot 9 \mathrm{H}_{2} \mathrm{O} \\
\mathrm{Al}(\mathrm{HCOO})_{3} \cdot 4 \mathrm{H}_{2} \mathrm{O}\end{array}$ & $\begin{array}{l}\text { PROLABO, min. } 98 \% \\
\text { Sintetizado en nuestro laboratorio a partir } \\
\text { del isopropóxido de aluminio }\end{array}$ \\
\hline $\mathrm{Cr}_{2} \mathrm{O}_{3}$ & $\begin{array}{c}\mathrm{Cr}\left(\mathrm{NO}_{3}\right)_{3} .9 \mathrm{H}_{2} \mathrm{O} \\
\mathrm{Cr}_{3}\left(\mathrm{CH}_{3} \mathrm{COO}\right)_{7}(\mathrm{OH})_{2}\end{array}$ & $\begin{array}{c}\text { HCB Bélgica, para análisis } \\
\text { ALDRICH, min. } 99 \%\end{array}$ \\
\hline $\mathrm{MgO}$ & $\mathrm{Mg}\left(\mathrm{NO}_{3}\right)_{2} \cdot 6 \mathrm{H}_{2} \mathrm{O}$ & MERCK, min. 99\% \\
\hline $\mathrm{CaO}$ & $\mathrm{Ca}\left(\mathrm{CH}_{3} \mathrm{COO}\right)_{2} \cdot \mathrm{H}_{2} \mathrm{O}$ & PANREAC, $\min .99 \%$ \\
\hline $\mathrm{CeO}_{2}$ & $\mathrm{Ce}\left(\mathrm{NO}_{3}\right)_{3}$ & RHONE-POULENC, $99.5 \%$ \\
\hline $\mathrm{MnO}$ & $\mathrm{Mn}\left(\mathrm{CH}_{3} \mathrm{COO}\right)_{2} \cdot 4 \mathrm{H}_{2} \mathrm{O}$ & ALDRICH, min. 99\% \\
\hline $\mathrm{ZrO}_{2}$ & $\mathrm{ZrOCl}_{2} .8 \mathrm{H}_{2} \mathrm{O}$ & AVOCADO, min. $98 \%$ \\
\hline $\mathrm{SnO}_{2}$ & $\mathrm{SnCl}_{2} \cdot 2 \mathrm{H}_{2} \mathrm{O}$ & PANREAC, $\min .98 \%$ \\
\hline
\end{tabular}




\section{RESULTADOS Y DISCUCIÓN}

\subsection{Estudio del sistema $\mathrm{Y}_{3} \mathrm{~A}_{15}-\mathrm{xCr}_{\mathrm{x}} \mathrm{O}_{12}$, disolución sólida}

A través de los estudios mediante difracción de rayos $X$, se evidencia la formación de la fase granate a partir de los $800^{\circ} \mathrm{C}$. Como se observa en la figura 2 aparece una mayor intensidad en los picos de difracción a medida que aumenta el contenido de cromo en la disolución sólida, lo que podría indicar un efecto de estabilización estructural por parte de dicho elemento. Dicho efecto ya se había comprobado en estudios previos, trabajando en la serie de disoluciones sólidas de granates de YAG-uvarovita (26). También hay que indicar que en los espectros de infrarrojos, figura 3 , se ha podido observar un aumento de intensidad de las bandas asignadas a las vibraciones correspondientes a la estructura granate, con el aumento del contenido de cromo.

Por otra parte, en los espectros de ultravioleta y visible de las muestras calcinadas a $600^{\circ} \mathrm{C}$, figura 4 , se observa una

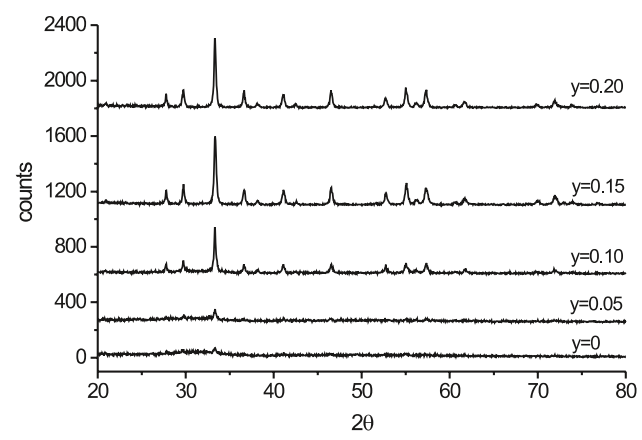

Fig. 2 Difractogramas de las muestras de la serie $\mathrm{Y}_{3} \mathrm{Al}_{5-\mathrm{y}} \mathrm{Cr}_{\mathrm{y}} \mathrm{O}_{12}$ sintetizadas a $800^{\circ} \mathrm{C}$

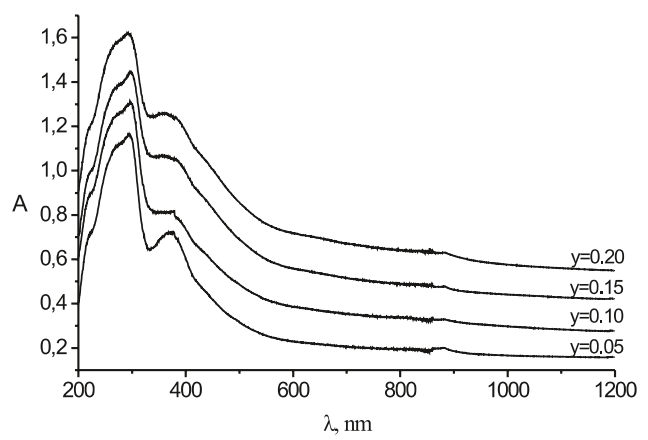

Fig. 4 Espectros de absorción en UV-Vis de las muestras de la serie $\mathrm{Y}_{3} \mathrm{Al}_{5-\mathrm{y}} \mathrm{Cr}_{\mathrm{y}} \mathrm{O}_{12}$ sintetizadas a $600^{\circ} \mathrm{C}$

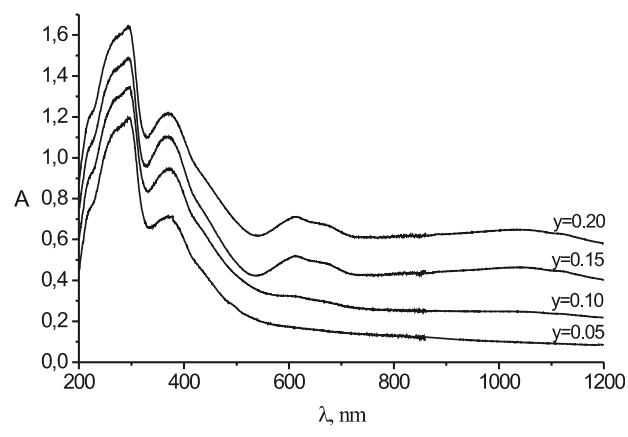

Fig. 6 Espectros NUV/Vis/NIR de las muestras de la serie $\mathrm{Y}_{3} \mathrm{Al}_{5-\mathrm{y}} \mathrm{Cr}_{\mathrm{y}} \mathrm{O}_{12}$ sintetizadas a $1200^{\circ} \mathrm{C}$ banda de transferencia de carga de máximo situado alrededor de $370 \mathrm{~nm}$ asignada a la presencia de cromo (VI). Como se puede ver en la figura 5, en las muestras de $y=0.15$ e $y=0.20$ calcinadas a $800^{\circ} \mathrm{C}$, aparte de dicha banda debida a cromo(VI), aparece una banda asignada a la presencia de cromo (IV), con máximo por encima de $1000 \mathrm{~nm}$, y una de cromo (III), con máximo a $610 \mathrm{~nm}$. Al último se atribuye la tonalidad verdosa de estas dos muestras. En cambio las otras dos muestras que contienen prácticamente sólo cromo en estado de oxidación seis son amarillas.

Con el fin de poder eliminar las interferencias que podría ocasionar la formación de estados de oxidación del cromo superiores a tres, se ha calcinado toda la serie a $1200^{\circ}$ y $1400^{\circ} \mathrm{C}$. Se puede observar en los correspondientes espectros que a $1200^{\circ} \mathrm{C}$, todavía persisten las bandas del cromo (IV) y (VI) (figura 6). Sin embargo, a $1400^{\circ} \mathrm{C}$, como se puede ver en la figura 7 , desaparece prácticamente la banda del $\mathrm{Cr}(\mathrm{VI})$ a $270 \mathrm{~nm}$ y se mantiene la del $\mathrm{Cr}(\mathrm{IV})$ a $1000 \mathrm{~nm}$, además de las correspondientes al $\mathrm{Cr}(\mathrm{III})$.

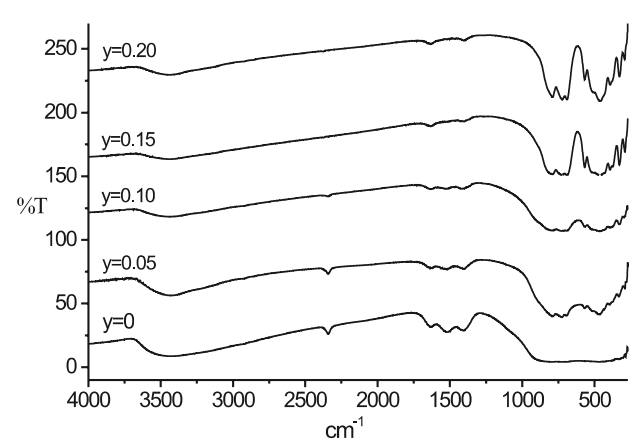

Fig. 3 Espectros de infrarrojos de las muestras de la serie $\mathrm{Y}_{3} \mathrm{Al}_{5-\mathrm{y}} \mathrm{Cr}_{\mathrm{y}} \mathrm{O}_{12}$ sintetizadas a $800^{\circ} \mathrm{C}$

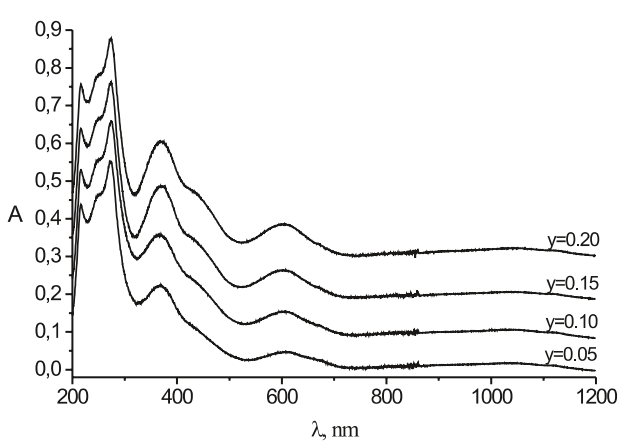

Fig. 5 Espectros de absorción en UV-Vis de las muestras de la serie $\mathrm{Y}_{3} \mathrm{Al}_{5-\mathrm{y}} \mathrm{Cr}_{\mathrm{y}} \mathrm{O}_{12}$ sintetizadas a $800^{\circ} \mathrm{C}$

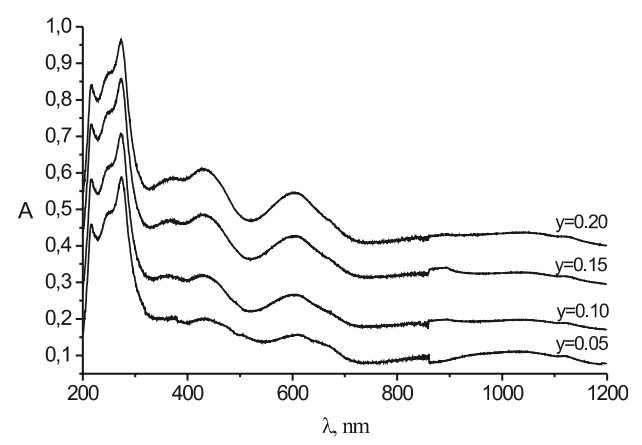

Fig. 7 Espectros NUV/Vis/NIR de las muestras de la serie $\mathrm{Y}_{3} \mathrm{Al}_{5-\mathrm{y}} \mathrm{Cr}_{\mathrm{y}} \mathrm{O}_{12}$ sintetizadas a $1400^{\circ} \mathrm{C}$ 
Además, no se observa en dichos espectros el desplazamiento de las bandas del $\mathrm{Cr}$ (III) hacia regiones más energéticas del espectro, en dirección a la zona del ultravioleta, tal como se desearía.

En la tabla III, se indican los valores de las coordenadas cromáticas y la intensidad del campo cristalino obtenido para la presente serie de disoluciones sólidas.

\subsection{Estudio del sistema $\mathrm{Y}_{3}-\mathrm{y}(\mathrm{M}, \mathrm{N}) \mathrm{yAl} \mathrm{I}_{5}-\mathrm{xCr}_{\mathrm{x}} \mathrm{O}_{12}$ $\mathrm{M}=\mathrm{Mn}(\mathrm{II}), \mathrm{Mg}(\mathrm{II}) ; \mathrm{N}=\mathrm{Ca}(\mathrm{II}), \mathrm{Ce}(\mathrm{IV})$, disolución sólida.}

En esta serie, la difracción de rayos $\mathrm{X}$ indica que también se obtiene una fase pura de granate siendo los difractogramas de las muestras calcinadas a partir de $800^{\circ} \mathrm{C}$ muy parecidos a los de la serie anterior (ver la figura 2).

Mediante la espectrofotometría ultravioleta y visible, se observa que la evolución de las muestras es muy parecida a la anterior serie de disoluciones sólidas estudiada. Como se puede ver en la figura 8 correspondiente a las muestras calcinadas a $1400^{\circ} \mathrm{C}$, aquí también la banda del cromo(VI) que se observa a más bajas temperaturas de calcinación entorno a $370 \mathrm{~nm}$, está reducida plenamente. Sin embargo, hay que resaltar que la banda asignada al $\mathrm{Cr}(\mathrm{IV})$ y cuyo máximo se sitúa hacia los $1000 \mathrm{~nm}$, aparece ligeramente más intensa. Este hecho podría ser explicado por la existencia de mayor concentración de $\mathrm{Cr}(\mathrm{IV})$, comparando con la serie anterior, provocado por la compensación de carga a través de la presencia de los iones divalentes en la presente serie.
También hay que resaltar que en esta serie tampoco parece observarse un desplazamiento de las bandas del $\mathrm{Cr}$ (III) hacia valores de longitudes de onda más bajos, como cabría esperar al sustituir el itrio por iones más pequeños, ver la tabla IV. La explicación aquí, como en el caso anterior, estaría en las distorsiones estructurales provocadas por la presencia del Cr(IV), como también lo puede confirmar el desdoblamiento de las bandas del $\mathrm{Cr}$ (III) que aparecen en la región del visible del espectro, aunque también podría deberse dicho desdoblamiento a un solapamiento de bandas del $\mathrm{Cr}(\mathrm{IV})$, que aparecen también en esa misma región.

Sin embargo, para la disolución sólida que contiene el granate YAG dopado con $\mathrm{Mg}$, Ce y $\mathrm{Cr}$, el campo cristalino es más elevado (tabla IV). En esta última disolución sólida no se detecta la presencia de $\mathrm{Cr}(\mathrm{IV})$, lo cual podría explicarse por un efecto de reducción ocasionado por el Ce (III) hacia dicho cromo según la ecuación: $\mathrm{Cr}^{4+}+\mathrm{Ce}^{4+} \rightarrow \mathrm{Cr}^{3+}+\mathrm{Ce}^{4+}$

\subsection{Estudio de las disoluciones sólidas $\mathrm{Y}_{3}-\mathrm{xMxA}_{15}-\mathrm{yCryO}_{12+0.5 \mathrm{x}}, \mathrm{M}=\mathrm{Zr}(\mathrm{IV}), \mathrm{Sn}(\mathrm{IV})$}

En la figura 9 se muestran los difractogramas de rayos $X$ de las muestras $\mathrm{Y}_{2} \mathrm{ZrAl}_{4.8} \mathrm{Cr}_{0.2} \mathrm{O}_{12+\delta}$ y $\mathrm{Y}_{2} \mathrm{SnAl}_{4.8} \mathrm{Cr}_{0.2} \mathrm{O}_{12+\delta}$ sintetizadas a $1400^{\circ} \mathrm{C}$. En ellos se puede apreciar la formación de fases secundarias importantes por su intensidad. Así, en las disoluciones sólidas dopadas con circonio y con estaño se obtienen los compuestos no estequiométricos $\mathrm{Y}_{0.15} \mathrm{Zr}_{0.85} \mathrm{O}_{1.93}$ y $\mathrm{Y}_{2} \mathrm{Sn}_{2} \mathrm{O}_{7}$, respectivamente, además de formarse una pequeña cantidad de $\alpha-\mathrm{Al}_{2} \mathrm{O}_{3}$, corindón.
TAbla III Parámetros ópticos de las MUESTRAS DE LA SERIE $\mathrm{Y}_{3} \mathrm{Al}_{5-\mathrm{y}} \mathrm{Cr}_{\mathrm{y}} \mathrm{O}_{12}(\mathrm{y}=0.05,0.10,0.15,0.20)$ CALCINADAS A $1400^{\circ} \mathrm{C}$ COMPARADOS CON LOS DE UNA ESMERALDA NATURAL

\begin{tabular}{|c|c|c|c|c|c|c|c|c|}
\hline $\begin{array}{c}\text { Composición } \\
\text { nominal }\end{array}$ & Color & $\mathrm{L}^{*}$ & $a^{*}$ & $\mathrm{~b}^{*}$ & $\begin{array}{c}{ }^{4} \mathrm{~T}_{1 \mathrm{~g}} \\
\uparrow \\
{ }^{4} \mathrm{~A}_{2 \mathrm{~g}} \\
{ }^{\mathrm{n}} \mathrm{nm}\end{array}$ & $\begin{array}{c}\text { Mínimo } \\
\text { entre las } \\
\text { dos bandas } \\
\text { del Cr(III), } \\
\text { nm }\end{array}$ & $\begin{array}{c}{ }^{4} \mathrm{~T}_{2 \mathrm{~g}} \\
\uparrow \\
\uparrow \\
{ }^{4} \mathrm{~A}_{2 \mathrm{~g}} \\
\mathrm{~nm}\end{array}$ & $\begin{array}{l}\Delta_{0} \\
\mathrm{eV}\end{array}$ \\
\hline $\mathrm{Y}_{3} \mathrm{Al}_{5-\mathrm{y}} \mathrm{Cr}_{\mathrm{y}} \mathrm{O}_{12}$ & \multirow{5}{*}{$\begin{array}{l}\text { Tonalidades } \\
\text { del verde }\end{array}$} & & & & & & & \\
\hline$y=0.05$ & & 97.56 & -2.71 & 5.85 & 432.8 & 546.8 & 610.0 & 2.033 \\
\hline$y=0.10$ & & 97.22 & -5.68 & 6.97 & 423.6 & 522.0 & 606.8 & 2.043 \\
\hline$y=0.15$ & & 95.14 & -7.55 & 8.19 & 424.0 & 521.2 & 606.4 & 2.045 \\
\hline $\mathrm{y}=0.20$ & & 84.78 & -5.44 & 6.86 & 427.2 & 519.6 & 606.4 & 2.045 \\
\hline $\begin{array}{c}\text { Esmeralda } \\
\text { natural }\end{array}$ & Verde & 47.25 & -5.76 & 7.00 & - & 515.2 & 620.8 & 1.997 \\
\hline $\mathrm{Be}_{3} \mathrm{Al}_{2-x} \mathrm{Cr}_{x} \mathrm{Si}_{6} \mathrm{O}_{18}$ & & & & & & & & \\
\hline
\end{tabular}

TABla IV PARÁmETROS ÓPTICOS DE LAS MUESTRAS DE LA SERIE

$\mathrm{Y}_{2.95} \mathrm{Mn}_{0.05} \mathrm{Al}_{1.95} \mathrm{Cr}_{0.1} \mathrm{O}_{12}, \mathrm{Y}_{2.9} \mathrm{Mg}_{0.1} \mathrm{Al}_{4.9} \mathrm{Cr}_{0.1} \mathrm{O}_{12}, \mathrm{Y}_{2.93} \mathrm{Mg}_{0.04} \mathrm{Ca}_{0.03} \mathrm{Al}_{4.98} \mathrm{Cr}_{0.02} \mathrm{O}_{12}$ y $\mathrm{Y}_{2.8} \mathrm{Mg}_{0.1} \mathrm{Ce}_{0.1} \mathrm{Al}_{4.8} \mathrm{Cr}_{0.2} \mathrm{O}_{12}$ SINTETIZADAS A $1400^{\circ} \mathrm{C}$

\begin{tabular}{|c|c|c|c|c|c|c|c|c|}
\hline Composición nominal & Color & $L^{*}$ & $a^{*}$ & $\mathrm{~b}^{*}$ & $\begin{array}{c}{ }^{4} \mathrm{~T}_{1 \mathrm{~g}} \\
\uparrow \\
{ }^{4} \mathrm{~A}_{2 \mathrm{~g}} \\
\mathrm{~nm}\end{array}$ & $\begin{array}{c}\text { Mínimo } \\
\text { entre las } \\
\text { dos } \\
\text { bandas } \\
\text { del } \\
\text { Cr(III), } \\
\text { nm }\end{array}$ & $\begin{array}{c}{ }^{4} \mathrm{~T}_{2 \mathrm{~g}} \\
\uparrow \\
{ }^{4} \mathrm{~A}_{2 \mathrm{~g}} \\
\mathrm{~nm}\end{array}$ & $\begin{array}{l}\Delta_{0} \\
\mathrm{eV}\end{array}$ \\
\hline $\mathrm{Y}_{2.95} \mathrm{Mn}_{0.05} \mathrm{Al}_{4.9} \mathrm{Cr}_{0.1} \mathrm{O}_{12}$ & $\begin{array}{l}\text { Marrón } \\
\text { verdoso }\end{array}$ & 79.34 & -4.84 & 7.74 & 491.6 & 543.2 & 616.8 & 2.010 \\
\hline $\mathrm{Y}_{2,9} \mathrm{Mg}_{0.1} \mathrm{Al}_{4,9} \mathrm{Cr}_{0.1} \mathrm{O}_{12}$ & Verde & 82.26 & -3.41 & 9.54 & 418.0 & 546.0 & 616.0 & 2.013 \\
\hline $\mathrm{Y}_{2.93} \mathrm{Mg}_{0.04} \mathrm{Ca}_{0.03} \mathrm{Al}_{4.98} \mathrm{Cr}_{0.02} \mathrm{O}_{12}$ & Marrón & 75.65 & 3.55 & 15.43 & 446.0 & 573.6 & 616.4 & 2.012 \\
\hline $\mathrm{Y}_{2.8} \mathrm{Mg}_{0.1} \mathrm{Ce}_{0.1} \mathrm{Al}_{4.8} \mathrm{Cr}_{0.2} \mathrm{O}_{12}$ & $\begin{array}{l}\text { Amarillo } \\
\text { verdoso }\end{array}$ & 79.04 & -5.04 & 20.92 & 455.6 & 543.2 & 600.4 & 2.065 \\
\hline
\end{tabular}

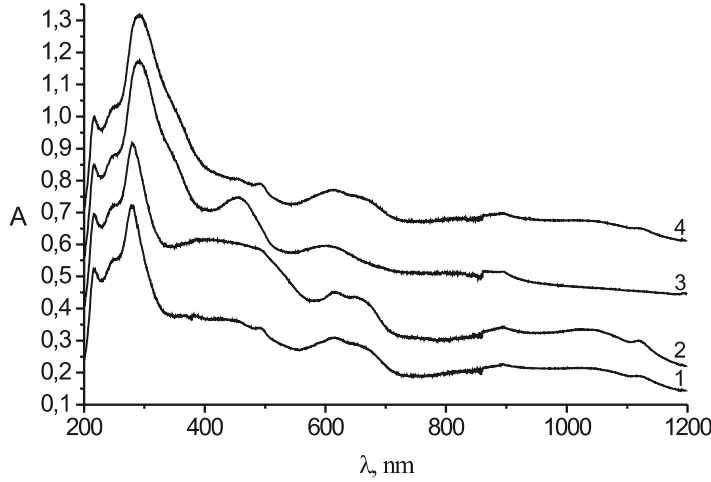

Fig. 8 Espectros NUV/Vis/NIR de las muestras $\mathrm{Y}_{2.9} \mathrm{Mg}_{0.1} \mathrm{Al}_{4.9} \mathrm{Cr}_{0.1} \mathrm{O}_{12}(1), \mathrm{Y}_{2.93} \mathrm{Mg}_{0.04} \mathrm{Ca}_{0.03} \mathrm{Al}_{4.98} \mathrm{Cr}_{0.02} \mathrm{O}_{12}(2)$ $\mathrm{Y}_{2.8} \mathrm{Mg}_{0.1} \mathrm{Ce}_{0.1} \mathrm{Al}_{4.8} \mathrm{Cr}_{0.2} \mathrm{O}_{12}(3) \mathrm{Y}_{2.95} \mathrm{Mn}_{0.05} \mathrm{Al}_{1.95} \mathrm{Cr}_{0.1} \mathrm{O}_{12}(4)$ sintetizadas a $1400^{\circ} \mathrm{C}$

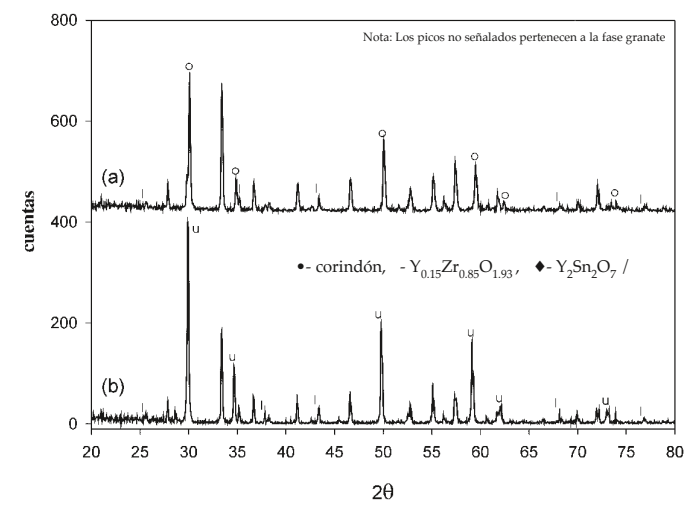

Fig. 9 Difractogramas de las muestras de composiciones nominales $\mathrm{Y}_{2} \mathrm{ZrAl}_{4.8} \mathrm{Cr}_{0.2} \mathrm{O}_{12}$ (a) y $\mathrm{Y}_{2} \mathrm{SnAl}_{4.8} \mathrm{Cr}_{0.2} \mathrm{O}_{12}$ (b) sintetizadas a $1400^{\circ} \mathrm{C}$ 
A través de los espectros de ultravioleta y visible de las muestras, figura 10, se observa el desplazamiento de las bandas de absorción acompañándose de un aumento de la intensidad de dichas bandas y desarrollándose una tonalidad rojo-violeta. En dichas muestras, el valor de $\Delta_{0}$ para el cromo(III) es bastante elevado, observándose en la muestra de YAG dopado con estaño y cromo un valor más alto que el obtenido para una muestra de rubí (tabla V).

Con el fin de identificar claramente las especies laterales formadas, correlacionar el valor del campo cristalino obtenido y poder asignar así correctamente la tonalidad obtenida, se han preparado y sintetizado dichas fases aparte, descartando la influencia que la fase corindón haya tenido en estos parámetros, debido a la baja intensidad de los picos de difracción asignados a dicha fase en los difractogramas.

Así, se ha preparado vía cerámica la disolución sólida $\mathrm{Y}_{2} \mathrm{Sn}_{1.95} \mathrm{Cr}_{0.05} \mathrm{O}_{7}$, después de calcinar a $1400^{\circ} \mathrm{C}$ la mezcla de precursores. En la figura 11 se muestra el correspondiente difractograma de rayos $X$, en el cual se puede apreciar la formación de única fase, correspondiendo todos los picos a un mismo compuesto, el pirocloro $\mathrm{Y}_{2} \mathrm{Sn}_{2} \mathrm{O}_{7}$.

La comparación de los espectros de absorción entre dicha fase y la de la composición nominal $\mathrm{Y}_{2} \mathrm{SnAl}_{4.8} \mathrm{Cr}_{0.2} \mathrm{O}_{12+\mathrm{d} \delta}$ fig. 12, indican poca variación y simplemente hay una ligera variación en la intensidad de los máximos de las bandas y presenta la muestra sintetizada una tonalidad roja-violácea bastante intensa, así como se indica en la tabla VI.

Este hecho puede indicar el que realmente la mayor aportación para la tonalidad la tiene la disolución sólida del cromo en el óxido $\mathrm{Y}_{2} \mathrm{Sn}_{2} \mathrm{O}_{7}$ y no en la estructura del granate.

\subsection{Pruebas de esmaltación}

Se han probado las muestras sintetizadas que presentaban tonalidades más intensas, como pigmentos para colorear bases cerámicas. Así, se han analizado las coordenadas cromáticas CIE Lab de las plaquetas esmaltadas y cocidas y, los resultados se indican en la tabla VII. De esta forma, se han podido comparar con las coordenadas cromáticas que presentan los correspondientes pigmentos sin introducirlos en ningún esmalte. Así, se puede ver que al esmaltar el pigmento de composición nominal $\mathrm{Y}_{2} \mathrm{SnAl}_{4.8} \mathrm{Cr}_{0.2} \mathrm{O}_{12+\delta}$ con la frita seleccionada, sus coordenadas $L^{*}$ (luminosidad) y a* (color rojo) no cambian, mientras que la coordenada $b^{*}$ se modifica ligeramente, indicando un pequeño aumento en la componente amarilla del color.

En el caso de la muestra de $\mathrm{Y}_{2} \mathrm{Sn}_{1.95} \mathrm{Cr}_{0.05} \mathrm{O}_{7}$, la coordena$\mathrm{da}^{*} \mathrm{a}^{*}$ aumenta, pero la coordenada $\mathrm{b}^{*}$ disminuye, indicando así un aumento en la componente azul. Esto se debe a la reducción de la absorción en el azul, la cual es debida a la transición ${ }^{4} \mathrm{~A}_{2 g} \rightarrow{ }^{4} \mathrm{~T}_{1 \mathrm{~g}}$ del cromo(III).

\section{CONCLUSIONES}

Se ha demostrado que en las condiciones de síntesis empleadas el cromo entra en la estructura del granate YAG tanto en estado trivalente como en estado tetravalente y hexavalente sin la adición especial de compensadores de carga,. La compensación de carga se realiza, probablemente, a través de la formación de vacantes.

El cromo(VI) se reduce prácticamente al calcinar las muestras durante 4 horas a $1400{ }^{\circ} \mathrm{C}$. Sin embargo el cromo(IV) persiste aún a esta temperatura tan elevada.

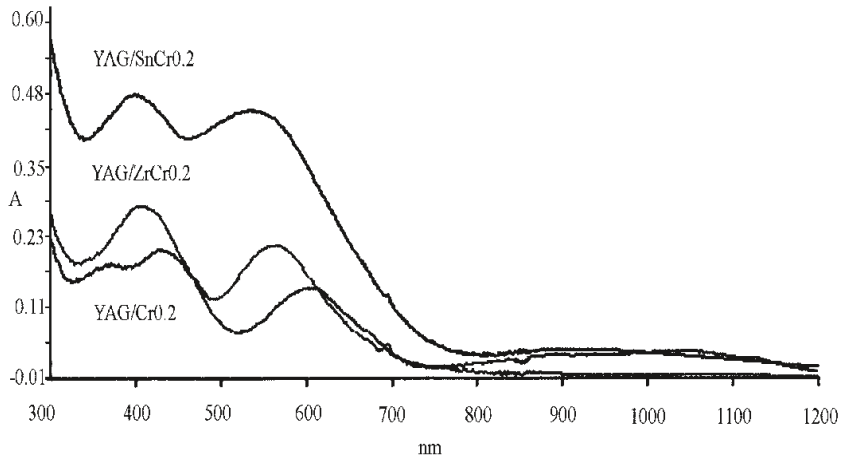

Fig.10 Espectros NUV/Vis / NIR de las muestras de composiciones nominales $\mathrm{Y}_{3} \mathrm{Al}_{4.8} \mathrm{Cr}_{0.2} \mathrm{O}_{12}, \mathrm{Y}_{2} \mathrm{ZrAl}_{4.8} \mathrm{Cr}_{0.2} \mathrm{O}_{12+\mathrm{d}}$ y $\mathrm{Y}_{2} \mathrm{SnAl}_{4.8} \mathrm{Cr}_{0.2} \mathrm{O}_{12+\mathrm{d}}$ sintetizadas a $1400^{\circ} \mathrm{C}$

TABLA V PARÁMETROS ÓPTICOS DE LAS MUESTRAS DE LA SERIE $\mathrm{Y}_{3-\mathrm{x}} \mathrm{M}_{\mathrm{x}} \mathrm{Al}_{5-\mathrm{y}} \mathrm{Cr}_{\mathrm{y}} \mathrm{O}_{12+0.5 \mathrm{x}} \mathrm{M}=\mathrm{Zr}(\mathrm{IV}), \mathrm{Sn}(\mathrm{IV})$ CALCINADAS A $1400^{\circ} \mathrm{C}$, COMPARADOS CON LOS DE RUBí NATURAL Y SINTÉTICO

\begin{tabular}{|c|c|c|c|c|c|c|c|c|}
\hline $\begin{array}{l}\text { Composición } \\
\text { nominal }\end{array}$ & Color & $\mathrm{L}^{*}$ & $\mathrm{a}^{*}$ & $b^{*}$ & $\begin{array}{c}{ }^{4} \mathrm{~T}_{1 \mathrm{~g}} \\
\uparrow \uparrow \\
{ }^{4} \mathrm{~A}_{2 \mathrm{~g}} \\
\mathrm{~nm}\end{array}$ & \begin{tabular}{|c} 
Mínimo \\
entre las \\
dos bandas \\
del Cr(III), \\
nm \\
\end{tabular} & $\begin{array}{c}{ }^{4} \mathrm{~T}_{2 \mathrm{~g}} \\
\uparrow \\
\uparrow \\
{ }^{4} \mathrm{~A}_{2 \mathrm{~g}} \\
\mathrm{~nm}\end{array}$ & $\begin{array}{l}\Delta_{0} \\
\mathrm{eV}\end{array}$ \\
\hline $\mathrm{Y}_{2} \mathrm{ZrAl}_{4,8} \mathrm{Cr}_{0.2} \mathrm{O}_{12+\delta}$ & $\begin{array}{l}\text { Rosa } \\
\text { pálido }\end{array}$ & 84.17 & 0.27 & 3.77 & 404.8 & 491.6 & 562.8 & 2.203 \\
\hline $\mathrm{Y}_{2} \mathrm{SnAl}_{4.8} \mathrm{Cr}_{0.2 \mathrm{O}} \mathrm{O}_{12+\delta}$ & Rojo & 68.35 & 9.78 & 2.06 & 400.4 & 458.0 & 538.0 & 2.305 \\
\hline $\begin{array}{l}\text { Rubí natural } \\
\mathrm{Al}_{2} \times \mathrm{Cr}_{\mathrm{x}} \mathrm{O}_{3}\end{array}$ & Rojo & 48.87 & 6.28 & 3.05 & 395.6 & 484.0 & 556.8 & 2.227 \\
\hline $\begin{array}{c}\text { Rubí sintet. vía } \\
\text { Pechini } \\
\mathrm{Al}_{1.92} \mathrm{Cr}_{0.08} \mathrm{O}_{3}\end{array}$ & Rojo & 74.88 & 7.98 & 0.06 & 407.6 & 480.0 & 562.0 & 2.206 \\
\hline
\end{tabular}

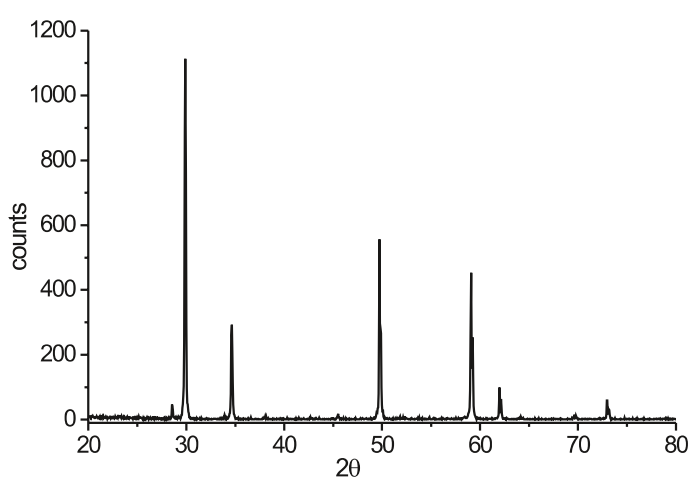

Fig. 11 Espectro de difracción de rayos $\mathrm{X}$ de la muestra de composición nominal $\mathrm{Y}_{2} \mathrm{Sn}_{1.95} \mathrm{Cr}_{0.05} \mathrm{O}_{7}$

TABLA VI PaRÁmetros ÓPticos de LA MUESTRA $\mathrm{Y}_{2} \mathrm{Sn}_{1.95} \mathrm{Cr}_{0.05} \mathrm{O}_{7}$ OBTENIDA VÍA CERÁMICA A $1400^{\circ} \mathrm{C}$, COMPARADOS CON LOS DE LA MUESTRA $\mathrm{Y}_{2} \mathrm{SnAl}_{4.8} \mathrm{Cr}_{0.2} \mathrm{O}_{12}$ Y DEL RUBí NATURAL

\begin{tabular}{|c|c|c|c|c|c|c|c|c|}
\hline $\begin{array}{l}\text { Composición } \\
\text { nominal }\end{array}$ & Color & $\mathrm{L}^{*}$ & $a^{*}$ & $b^{*}$ & $\begin{array}{c}{ }^{4} \mathrm{~T}_{1 \mathrm{~g}} \\
\uparrow \\
{ }^{4} \mathrm{~A}_{2 \mathrm{~g}} \\
\mathrm{~nm}\end{array}$ & $\begin{array}{c}\text { Minimo } \\
\text { entre las } \\
\text { dos bandas } \\
\text { del cromo, } \\
\mathrm{nm}\end{array}$ & $\begin{array}{c}{ }^{4} \mathrm{~T}_{2 g} \\
\uparrow \\
{ }^{4} \mathrm{~A}_{28} \\
\mathrm{~nm}\end{array}$ & $\begin{array}{l}\Delta_{0} \\
\text { eV }\end{array}$ \\
\hline $\mathrm{Y}_{2} \mathrm{Sn}_{1.95} \mathrm{Cr}_{0.05} \mathrm{O}_{7}$ & $\begin{array}{c}\text { Rojo- } \\
\text { viaoláceo }\end{array}$ & 51.50 & 12.38 & 1.52 & 385.6 & 443.6 & 518.8 & 2.390 \\
\hline $\mathrm{Y}_{2} \mathrm{SnAl}_{4.8} \mathrm{Cr}_{\mathrm{I}_{0.2}} \mathrm{O}_{12+\delta}$ & Rojo & 68.35 & 9.78 & 2.06 & 400.4 & 458.0 & 538.0 & 2.305 \\
\hline $\begin{array}{l}\text { Rubi natural } \\
\mathrm{Al}_{2-x} \mathrm{Cr}_{x} \mathrm{O}_{3}\end{array}$ & Rojo & 48.87 & 6.28 & 3.05 & 395.6 & 484.0 & 556.8 & 2.227 \\
\hline $\begin{array}{l}\text { Rubí sintet. via } \\
\text { Pechini } \\
\mathrm{Al}_{1.92} \mathrm{Cr}_{0.08} \mathrm{O}_{3}\end{array}$ & Rojo & 74.88 & 7.98 & 0.06 & 407.6 & 480.0 & 562.0 & 2.206 \\
\hline
\end{tabular}




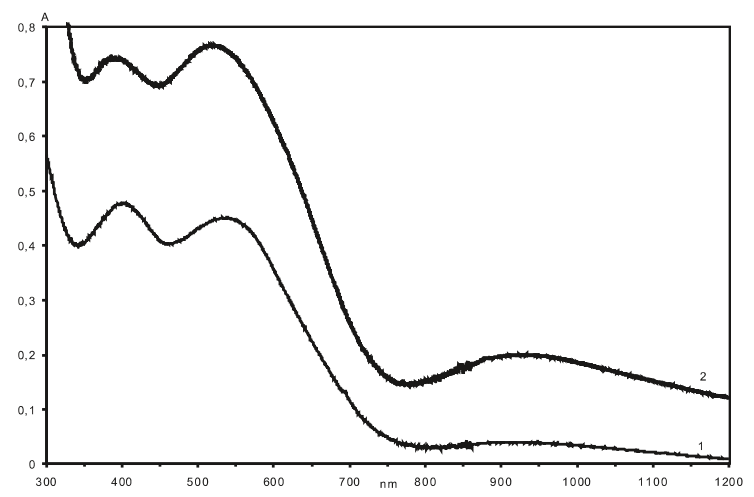

Fig.12 Espectros NUV/Vis/NIR de las muestras de composiciones nominales $\mathrm{Y}_{2} \mathrm{SnAl}_{4.8} \mathrm{Cr}_{0.2} \mathrm{O}_{12+\delta}(1) \mathrm{y}_{2} \mathrm{Sn}_{1.95} \mathrm{Cr}_{0.05} \mathrm{O}_{7}$ (2) sintetizadas a $1400^{\circ} \mathrm{C}$

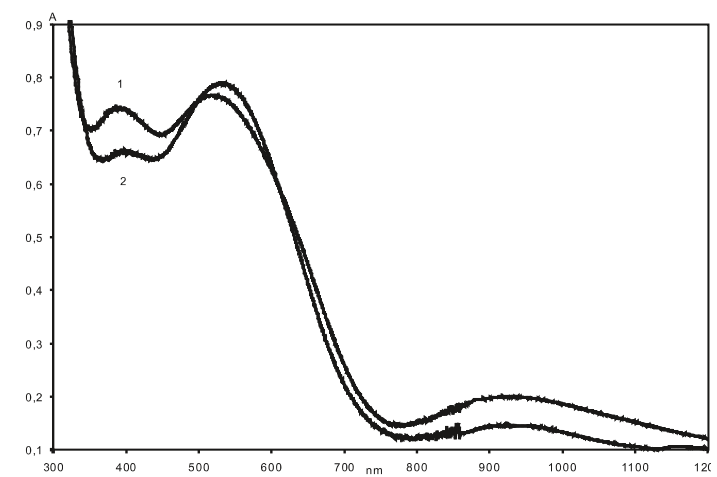

Fig. 13 Espectros NUV/Vis/NIR de la muestra $\mathrm{Y}_{2} \mathrm{Sn}_{1.95} \mathrm{Cr}_{0.05} \mathrm{O}_{7}(1)$ sintetizada a $1400^{\circ} \mathrm{C}$ y el esmalte coloreado con ella (2)

Se han intentado varios caminos para aumentar la intensidad del campo cristalino que actúa sobre el $\mathrm{Cr}(\mathrm{III})$ en la estructura del YAG, en un entorno octaédrico, para poder así hacer evolucionar la tonalidad verde a roja. La disminución de la concentración de cromo así como la introducción de iones divalentes, más pequeños que el itrio, no han conducido al éxito. Esto se ha atribuido principalmente a la formación de cromo(IV) que provoca distorsiones en la propia estructura del granate.

Sólamente la introducción de elementos tetravalentes (circonio y estaño) provoca transición de la tonalidad verde a la roja. En este último caso la falta de elementos apropiados para compensar la carga exceciva del Zr(IV) o del Sn(IV), origina la formación de fases laterales. Se ha demostrado que en el caso de la introducción de estaño como agente dopante, se origina una fase secundaria $\mathrm{Y}_{2} \mathrm{Sn}_{2} \mathrm{O}_{7}$, la cual es responsable, de forma importante, de la tonalidad roja observada.

Para contrastar esto, se ha sintetizado un pigmento de composición $\mathrm{Y}_{2} \mathrm{Sn}_{1.95} \mathrm{Cr}_{0.05} \mathrm{O}_{7}$, que presenta buena estabilidad térmica y una tonalidad roja-violácea. Al introducirlo en una frita transparente de cinc, la componente azul de dicha tonalidad ha aumentado.

\section{AGRADECIMIENTOS}

Radostin Pavlov agradece la Agencia Española de Cooperación Internacional (AECI) por la beca de investigación que tiene concedida.
TABLA VII PARÁMETROS ÓPTICOS DE LOS PIGMENTOS DE COMPOSICIONES NOMINALES $\mathrm{Y}_{3} \mathrm{Al}_{4.8} \mathrm{Cr}_{0.2} \mathrm{O}_{12}, \mathrm{Y}_{2} \mathrm{SnAl}_{4.8} \mathrm{Cr}_{0.2} \mathrm{O}_{12}, \mathrm{Y}_{2} \mathrm{Sn}_{1.95} \mathrm{Cr}_{0.05} \mathrm{O}_{7}$ OBTENIDOS A $1400^{\circ} \mathrm{C}$, COMPARADOS CON LOS DE LOS ESMALTES COLOREADOS CON LOS MISMOS.

\begin{tabular}{|c|c|c|c|c|c|c|c|c|}
\hline $\begin{array}{l}\text { Composición } \\
\text { nominal }\end{array}$ & Color & $\mathrm{L}^{*}$ & $a^{*}$ & $b^{*}$ & $\begin{array}{c}{ }^{4} \mathrm{~T}_{1 \mathrm{~g}} \\
\uparrow \\
{ }^{4} \mathrm{~A}_{2 \mathrm{~g}} \\
\mathrm{~nm}\end{array}$ & $\begin{array}{c}\text { Minimo } \\
\text { entre las } \\
\text { dos bandas } \\
\text { del Cr(III), } \\
\text { nm }\end{array}$ & $\begin{array}{c}{ }^{4} \mathrm{~T}_{2 \mathrm{~g}} \\
\uparrow \\
\uparrow \\
{ }^{4} \mathrm{~A}_{2 \mathrm{~g}} \\
\mathrm{~nm}\end{array}$ & $\begin{array}{l}\Delta_{0} \\
\mathrm{eV}\end{array}$ \\
\hline $\mathrm{Y}_{3} \mathrm{Al}_{4.8} \mathrm{Cr}_{0.2} \mathrm{O}_{12}$ & Verde & 84.78 & -5.44 & 6.86 & 427.2 & - & 606.4 & 2.045 \\
\hline $\begin{array}{c}\text { Esmalte de } \\
\mathrm{Y}_{3} \mathrm{Al}_{4.8} \mathrm{Cr}_{0.2} \mathrm{O}_{12}\end{array}$ & Verde & 82.57 & -3.38 & 14.97 & - & 537.6 & 585.2 & 2.119 \\
\hline $\mathrm{Y}_{2} \mathrm{SnAl}_{4.8} \mathrm{Cr}_{0.2} \mathrm{O}_{12+\delta}$ & Rojo & 68.35 & 9.78 & 2.06 & 400.4 & 458.0 & 538.0 & 2.305 \\
\hline $\begin{array}{c}\text { Esmalte con } \\
\mathrm{Y}_{2} \mathrm{SnAl}_{4.8} \mathrm{Cr}_{0.2} \mathrm{O}_{12+\delta}\end{array}$ & Rojo & 67.81 & 9.77 & 7.17 & - & 481.6 & 536.0 & 2.313 \\
\hline $\mathrm{Y}_{2} \mathrm{Sn}_{1,95} \mathrm{Cr}_{0.05} \mathrm{O}_{7}$ & $\begin{array}{c}\text { Rojo- } \\
\text { viaoláceo }\end{array}$ & 51.50 & 12.38 & 1.52 & 385.6 & 443.6 & 518.8 & 2.390 \\
\hline $\begin{array}{c}\text { Esmalte con } \\
\mathrm{Y}_{2} \mathrm{Sn}_{1.95} \mathrm{Cr}_{0.05} \mathrm{O}_{7}\end{array}$ & $\begin{array}{l}\text { Rojo- } \\
\text { violeta }\end{array}$ & 50.72 & 15.59 & -3.17 & 397.6 & 436.4 & 533.2 & 2.326 \\
\hline
\end{tabular}

\section{BIBLIOGRAFÍA}

1. M.Regueiro, E.Sánchez, V.Sanz y E.Criado, “Cerámica Industrial en España," Bol.Soc.Esp. Ceram.Vidr., 39(1) 5-30 (2000).

2. J.Portolés, J.Sánchez, C.Soler y D.Redondo, “Decoración del Grés Porcelánico Natural con Aplicaciones de Naturaleza Organometálica," ," Bol.Soc.Esp.Ceram.Vidr., 39(1) 63-73 (2000).

3. "Nuevos Productos y Tecnologías en la Fabricación de Esmaltes y Pigmentos Cerámicos," J.Carda, J.Alarcón y J.Rincón eds., Coeditan: Socieda Española de Cerámica y Vidrio y Faenza Editrice Ibérica S.A., Castellón (España), 1992

4. M.Llusar, “Optimizaión Medioambiental de la Síntesis de Pigmentos Cerámicos: Aplicación de Métodos Sol-Gel," Tesis Doctoral, Universitat Jaume I, Castellón (España), 1998.

5. F.Bondioli, T.Manfredini, "Pigmenti Inorganici: Progettazione, Produzione ed Aplicaczioni Industriali," Ceramica Informazione, 383 (11), 811-816 (1998).

6. S.Geller, "Crystal Chemistry of the Garnets," Z.Kristallogr., 125, 1-47 (1967).

7. F.P.Glasser, "On the Stabillity and Synthesis of Uvarovite $\mathrm{Ca}_{3} \mathrm{Cr}_{2} \mathrm{Si}_{3} \mathrm{O}_{12}$ " Am.Mineral., 44, 11-12 (1959).

4. J.Alarcón, P.Escribano, J.Cargallo, “ $\mathrm{Cr}_{2} \mathrm{O}_{3}-\mathrm{CaO}-\mathrm{SiO}_{2}$ Based Ceramic Pigments," Br.Ceram. Trans.J., 83, 81-83 (1984).

9. J.Carda, G.Monrós, P.Escribano, "Synthesis of Uvarovite Garnet" J.Am.Ceram.Soc., 72 (1) 160-62 (1989).

10. F.P.Glasser, E.F.Osborn, "Phase Equilibrium Studies in the System $\mathrm{CaO}-\mathrm{Cr}_{2} \mathrm{O}_{3}-\mathrm{SiO}_{2}$," J. Amer.Ceram.Soc., 41, 358-367 (1958).

11. Y.Kalisky, "Hosts for Solid-State Luminescent Systems," in "Wide-Gap Luminescent Materials: Theory and Applications," Edited by Stanley R.Rotman, Kluwer Academic Publishers, 1997.

12. A.Ikesue, K.Kamata, K.Yoshida, "Synthesis of $\mathrm{Nd}^{3+}, \mathrm{Cr}^{3+}$ - Codoped YAG Ceramics for High-Efficiency Solid-State Lasers," J.Am.Ceram.Soc., 78 (9) 2545-47 (1995).

13. D.Ravichandran, R.Roy, A.Chakovskoi, C.Hunt, W.White, S.Erdei, "Fabrication of $\mathrm{Y}_{3} \mathrm{Al}_{5} \mathrm{O}_{12}$ :Eu Thin Films and Powders for Field Emission Display Applications," J. Luminescence, 71, 291-297 (1997).

14. R.Rao, "Preparation and Characterization of Fine-Grain Yttrium-Based Phosphors by SolGel Process," J.Electrochem.Soc.,143 (1) 189-197 (1996).

15. R.Olazcuaga, Pigmentos Rojos con Elementos de Transición o Tierras Raras para Cerámica de Alta Temperatura," Capítulo V, pag. 93-109, Publicado en "Nuevos Productos y Tecnologías de Esmaltes y Pigmentos Cerámicos," J.Carda, J.Alarcón y J.Rincón eds., Coeditan: Socieda Española de Cerámica y Vidrio y Faenza Editrice Ibérica S.A., Castellón (España), 1992.

16. H.Eilers, U.Hommerich, S.M.Jacobsen, W.M.Yen, Phys.Rev. B 49, 15505 (1994).

17. S.Sugano, Y.Tanabe, J.Phys. Soc.Japan, 12, 556 (1957);13, 880 (1958); 13, 899 (1958).

18. L.E.Orgel, "Ion Compression and the Colour of Rubi," Nature, 179 (29) 1348, (1957).

19. H.Carstens, "The Red-Green Change in Chromium-Bearing Garnets,"Contr.Mineral. and Petrol. 41, 273-276 (1973).

20. A.Ikesue, K.Yoshida, K.Kamata, "Transparent $\mathrm{Cr}^{4+}$-Doped YAG Ceramics for Tunable Lasers," J.Am.Ceram.Soc., 79 (2) 507-509 (1996).

21. D.Reinen, "Structure and Bonding," Ed. Springer Verlag, Vol.6, pag.39 (1969).

22. M.P.Pechini, U.S.Patent No.3 330, 697, July (1967).

23. M.Kakihana, "Sol-Gel Preparation of High Temperature Superconducting Oxides," J.SolGel Sci.Tecnol., 6, 7-55 (1996).

24. M. Kakihana, M. Arima, Y. Nakamura, M. Yashima, M. Yoshimura, "Polimerized Complex Route to Barium Titanate Powders Using Barium-Titanium Mixed-Metal Citric Acid Complex," J.Am.Ceram.Soc., 79 (11) 2847-56 (1996).

25. M. Kakihana, M. Arima, Y. Nakamura, M. Yashima, M. Yoshimura, "Spectroscopic Characterization of Precursors Used in the Pechini-Type Polymerizable Complex Processing of Barium Titanate," Chem.Mater,,11, 438-450 (1999).

26. J.Andrés, A.Beltrán, J.Carda, J.Igualada, G.Monrós, M.Tena, "Estudio Teórico de la Estabilidad de la Disolución Sólida de Granates Uvarovita-YAG," Bol.Soc.Esp.Ceram. Vidr., 31 (3) 326-328 (1992)

Recibido: 18-9-99

Aceptado: 26-1-00 